\title{
Using Health-Seeking Pattern to Estimate Disease Burden from Sentinel Surveillance
}

\author{
Eric H.Y. Lau*, Qiqi Zhang, Kin On Kwok, Irene O. Wong, Dennis K. Ip and Benjamin J. \\ Cowling
}

School of Public Health, University of Hong Kong, Hong Kong, China

\section{Objective}

This study described health-seeking behavior of the general population specific to different symptoms, at different times of the year. This information allows the estimation of population disease burden over the year using sentinel surveillance data. We will use influenza-like illness (ILI) as an example.

\section{Introduction}

The general health-seeking behavior has been well described in different populations. However, how different symptoms have driven health-seeking behavior was less explored. From the patient's perspective, health-seeking behavior tends to be responsive to discomfort or symptoms rather than the type of diseases which is unknown before medical consultation, hence symptom-specific behavior may more realistically reflect responses from the public which is subsequently captured by syndromic surveillance.

In Hong Kong, sentinel surveillance of common diseases, such as ILI and acute diarrhoeal diseases, consists of general practitioners (GP), general outpatient clinics (GOPC) and Chinese medicine practitioners (CMP). These existing sources of syndromic surveillance data are affected by the choice of health services and health seeking behavior and hence may over- or under-represent actual disease burden. By understanding health-seeking behavior at different times of the year, we could estimate the disease burden in the population, and population subgroup from multiple surveillance data.

\section{Methods}

We conducted 4 rounds of longitudinal randomized telephone surveys over 1.5 year, to describe symptom-specific health-seeking behavior at times with different level of disease activity (Figure 1). We collected information if healthcare service was being sought for and types of medical consultation after onset of different symptoms. The information were further utilized to estimate the ILI burden in specific age groups, by the following relation:

$$
\begin{aligned}
& \mathrm{k}_{1} \mathrm{~s}_{1}=\mathrm{c}_{11} \mathrm{~N}_{1}+\mathrm{c}_{12} \mathrm{~N}_{2}+\mathrm{c}_{13} \mathrm{~N}_{3} \\
& \mathrm{k}_{2} \mathrm{~s}_{2}=\mathrm{c}_{21} \mathrm{~N}_{1}+\mathrm{c}_{22} \mathrm{~N}_{2}+\mathrm{c}_{23} \mathrm{~N}_{3} \\
& \mathrm{k}_{3} \mathrm{~s}_{3}=\mathrm{c}_{31} \mathrm{~N}_{1}+\mathrm{c}_{32} \mathrm{~N}_{2}+\mathrm{c}_{33} \mathrm{~N}_{3}
\end{aligned}
$$

where $s_{i}, k_{i}, c_{i,}$ and $N_{i}$ represent ILI consultation rates from sentinel surveillance of health service type $i$, scaling factor from surveillance data to the population with ILI seeking health service type $i$, likelihood of consultation to health service type $i$ for age group $j$ with ILI, and the population size of age group $j$ with ILI. From the longitudinal survey, we obtained estimates for $c_{i j}$ and $k_{i}$ during the survey period. Using the surveillance data from GP, GOPC and CMP, we obtained estimates $N_{i}$ by solving the above relations.

\section{Results}

Fever is the symptom most likely to prompt people to seek medical attention. We further focused on ILI, defined as fever $\geq 37.8$ plus either cough or sore throat. There were 111 episodes of ILI in the study period. Of which, $85.6 \%, 6.3 \%$ and $18.9 \%$ visits GP, GOPC and CMP respectively (some have sought for multiple health service types), while $9 \%$ did not seek for any medical attention. Based on the relation in the Methods, we estimated the weekly age-specific ILI burden over time from GP, GOPC and CMP surveillance (Figure 2).
The estimated ILI burden for the population aged $\leq 15$ and $\geq 55$ years captured the peak in February-March in 2015 from surveillance of ILI institutional outbreaks (e.g. schools, elderly care centers). However, no such increased ILI burden was observed in the 16-54 years age group in the period.

\section{Conclusions}

Syndromic surveillance data from different sources (e.g. medical consultation or google flu trends) were affected by different health seeking or reporting behavior. By understanding and quantifying these behaviors at different times, it is possible to estimate disease burden in the population.

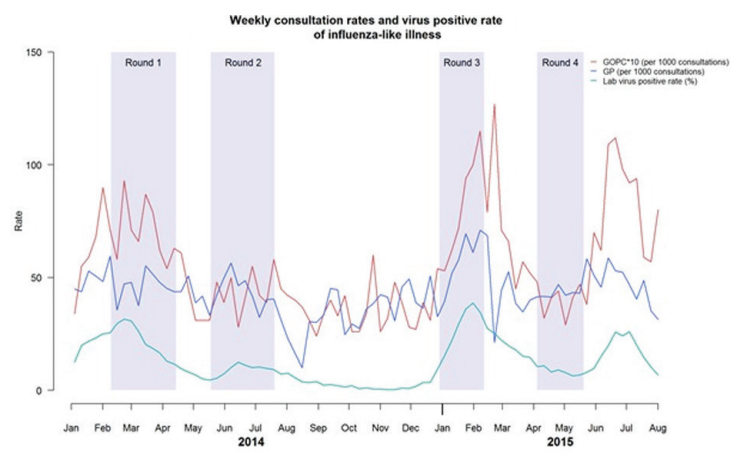

Timing covered by the 4 rounds of survey

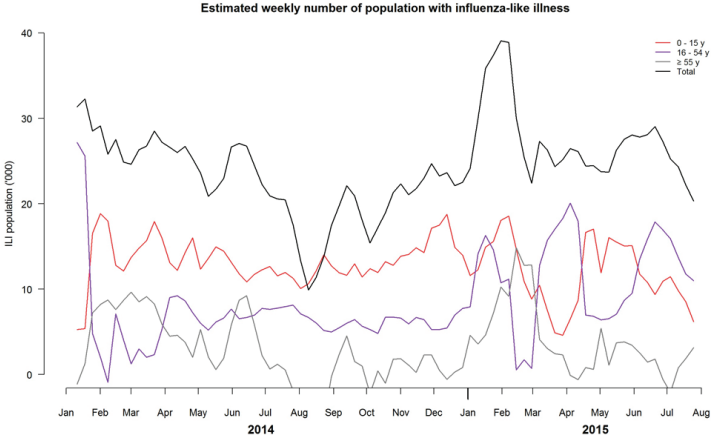

Estimated weekly age-specific burden of ILI

\section{Keywords}

Health seeking behaviour; symptoms; influenza like illness; sentinel surveillance

\section{Acknowledgments}

This work is supported by the Area of Excellence Scheme of the Hong Kong University Grants Committee, and the Health and Medical Research Fund of the Food and Health Bureau, Government of the Hong Kong Special Administrative Region.

\section{${ }^{\star}$ Eric H.Y. Lau}

E-mail: ehylau@hku.hk 